\title{
Osteoma of mastoid process obstructing external auditory canal: A case report
}

\author{
Aziz Mustafa \\ ENT Clinic, University Clinical Center of Kosova, Prishtina, Kosovo; aziz_mustafa2000@yahoo.com
}

Received 21 January 2012; revised 19 February 2012; accepted 5 March 2012

\begin{abstract}
Objective: To discuss the surgical treatment of recurrent osteoma of the mastoid process of the temporal bone, that obstructed external auditory canal causing unilateral conductive hearing loss. Setting: The study was carried out in ENT Clinic, University Clinical Center of Kosovo, Prishtina, Kosovo. Design: Retrospective review of a clinical case. Patient, Intervention and Result: We treated surgically with success a 14-year-old boy with osteoma of mastoid process, obstructing the external auditory canal of the left ear and causing conductive hearing loss. Axial and coronal computed tomography scans revealed an compact bone lesion that obstructed the canal. Complete removal of the lesion was achi- eved by a retroauricular approach. Conclusion: In order to achieve complete removal of the osteoma, drilling of the lesion must be performed not through the tumor, but around the osteoma, in the surrounding bone tissue.
\end{abstract}

Keywords: Mastoid Process Osteoma; Temporal Bone Tumors; Surgical Treatment; Case Report

\section{INTRODUCTION}

Osteomas can develop in every part of the temporal bone, being mostly asymptomatic. In the paranasal sinuses it is the most frequent benign tumor, occurring often in frontal and ethmoidal sinus, but it is rare in temporal bone. They present an average incidence of $1 \%$ $3 \%$ and are most commonly found in young and middleaged males according to most recent publications. Histologically, mastoid osteoma is classified as osteoid tissue included in osteoblastic tissue and surrounded by reactive bone. Three types of mastoid osteomas can be distinguished: compact osteomas, spongy osteomas and those with mixed characteristics [1,2]. In symptomatic cases, diagnosis can be suspect by x-ray or better by CT scan and confirmed by histopathology exam. Treatment of the osteoma is surgical, but in cases with petrous apex and middle ear involvement, the surgery can be challenging.

\section{CASE PRESENTATION}

A 14-year-old boy referred to our center from a regional hospital after two previous surgical procedures. Main complaint, in the first presentation to local ENT specialist was left side mild hearing loss. Otoscopy showed narrowing of the left external auditory canal (EAC). Pure tone audiometry (PTA) revealed mild conductive hearing loss of $45 \mathrm{~dB}$ in frequencies 500, 1000 and $2000 \mathrm{~Hz}$, with complete saved air bone gap (ABG). A CT scan was performed and showed an irregular hyperdense formation of the left mastoid process, with dimensions $2.3 \times 2$ $\times 2.6 \mathrm{~cm}$, narrowing and closing nearly the entire EAC. A local anesthesia surgical procedure was performed, drilling the protruding part of the osteoma in the posterior wall of EAC. The canal than was dressed with gauze tampon. Despite this procedure, the bone continued to growth and the canal was closed again. The procedure of the superficial drilling was repeated after 2 months, but without any success: the bone continued to grow and the EAC was narrowed and closed again.

When the patient was referred to our center, the complaints were hearing loss and filling of fullness of the left ear. The otoscopy showed nearly completely closed left EAC, with thinned skin over the bone of the posterior wall. The PTA revealed again mild conductive hearing loss of $45 \mathrm{~dB}$ in frequencies 500, 1000 and $2000 \mathrm{~Hz}$, with complete saved air bone gap (ABG). A new CT scan was similar to the previous scan, showing hyperdense bone measured $2 \times 1.6 \times 0.9 \mathrm{~cm}$ (Figure 1). After preparation, a surgical procedure in local anesthesia was performed by the author. In a retroauricular approach, mastoid tip was exposed as for simple mastoidectomy. The osteoma was identified and the drilling around it was performed, caring for facial nerve. Gradually, the osteoma was removed completely, leaving a small defect on posterior wall that was covered with remaining skin. Removed tumor was send to the histopathology exam. 


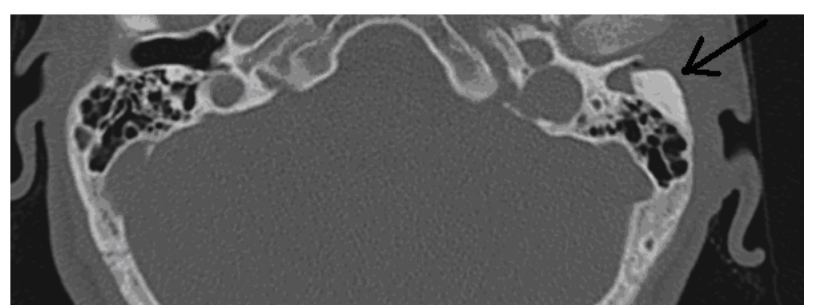

Figure 1. Axial view CT scan, with arrow showing left mastoid bone osteoma narrowing EAC.

The canal was dressed with gauze with antibiotic and corticoid unguent. The skin of the canal covered the defect for the next two weeks and the hearing was restored. Histopathology results of the removed bone shows compact bone tissue. One year after the surgery there is no sign of recurrence of disease, clinically nor radiological.

\section{DISCUSSION}

Osteomas in temporal bone are very rare benign osteogenic tumors that have been reported in literature in only 137 cases between 1861 and 2011 [1,3]. When they occur, they are seen most commonly in the external ear canal. Osteoma originating from the middle ear is very rare. Those from the mastoid are even rarer [4].

Osteomas are a slow growing neoplasm of unknown etiology. There are three theories discussing the etiology: congenital theory, based on facts that some osteomas occurs in male patients in puberty; infectious theory, reported in patients with recurrent suppurative otitis media; and traumatic theory, in cases with micro trauma of subperiostal hematoma [5].

Based on bone composition osteomas are divided into spongious (osteoma spongiosum), compact osteomas (osteoma eburnum) and mixed (osteoma mixta); based on growth direction, into outward-growing (exosteoma) and in inward-growing (endosteoma); into unilateral and bilateral; based on size, into small and gigantic; by surface structure, into smooth and multilobular; by number, into solitary and multiple and by symmetry into symmetrical and asymmetrical [6]. In our presented case the osteoma was unilateral, out-wards growing, composed from compact bone, middle sized, smooth-surfaced, solitary and asymmetrical.

Mastoid osteomas are usually asymptomatic and can be detected during otoscopy, but sometimes can be presented with different symptoms and signs, e.g. hearing loss, tinnitus, aural fullness, intermittent otalgia, retroauricular bone tumefaction, tympanic membrane perforation and suppurative drainage. In most cases, the diagnoses were confirmed by CT scan or visual inspection in surgical exploration. The lesion would appear as a well circumscribed mass with soft tissue density, egg-shell like density or bone density in CT scan [7]. In our case, the main clinical sign was unilateral conductive hearing loss. Diagnosis was confirmed by CT scan that showed a compact bone mass with high density.

Knowledge about the relationship between cholesteatoma and mastoid osteoma is scarce, but there are nevertheless theories that correlate both entities. Some authors postulate that external auditory canal osteomas may cause retention of epithelial residues and ear wax, and cause secondary cholesteatoma [8].

Treatment of the mastoid osteoma is surgical and it is indicated for osteomas that are symptomatic or cosmetically unacceptable. Excision or drilling of superficial lesions of the mastoid and squama is a simple procedure [4]. When osteomas become too large for en bloc remo$\mathrm{val}$, it becomes necessary to break the mass into smaller pieces [7]. In cases of mastoid osteomas extending into the fallopian canal and bony labyrinth, complete excision is not indicated since there may be damage to these structures. Follow up is needed in cases where partial excision is done or where expectant treatment is adopted [4]. If surgically properly removed, osteomas do not have tendencies of recurrence. In a series of 9 patients with osteomas in the different parts of the temporal bone, author Viswanatha doesn't notice recurrence or any complication during a period of one year of follow-up [9]. In our presented case, patient was treated surgically twice, via endaural approach, before coming in our institution, where the mastoid osteoma protruding to the EAC was completely removed with retroauricular approach. We consider that in previous surgical interventions, osteoma was treated partially only by drilling on the tumor tissue, in contrast with our approach that was drilling around the osteoma for successful complete removal.

\section{REFERENCES}

[1] Domínguez Pérez, A.D., Rodríguez Romero, R., Domínguez Durán, E., Riquelme Montaño, P., Alcántara Bernal, R. and Monreal Rodríguez, C. (2011) The mastoid osteoma, an incidental feature? Acta Otorrinolaringológica Española, 62, 140-143. doi:10.1016/0017-9310(75)90243-4

[2] El Fakiri, M., El Bakkouri, W., Halimi, C., Aït Mansour, A. and Ayache, D. (2011) Mastoid osteoma: Report of two cases. European Annals of Otorhinolaryngology, Head and Neck Diseases, 128, 266-268. doi:10.1016/j.anorl.2011.03.001

[3] Magliulo, G. and Pulice, G. (2005) Mastoid osteoma. Anales Otorrinolaringológicos Ibero-Americanos, 32, 271-278.

[4] Das, A.K. and Kashyap, R.C. (2005) Osteoma of the Mastoid Bone-A Case Report. MJAFI, 61, 86-87.

[5] Martinez, R., Morais, D., Ramirez, B., Martinez, P., Benito, J.I. (2003) Osteoma of the mastoid. Acta Otorrinolaringol Esp, 54, 94-97.

[6] Sente, M. (2009) External auditory canal osteoma. Srpski 
Arhiv za Celokupno Lekarstvo, 137, 73-76. doi:10.2298/SARH0902073S

[7] Kim, W.C., Oh, S.J., Kang, J.M. and Ahn, H.Y. (2006) Multiple osteomas of the middle ear. European Archives of Oto-Rhino-Laryngology, 263, 1151-1154. doi:10.1007/s00405-006-0123-X

[8] Lee, D.H., Jun, B.C., Park, C.S. and Cho, K.J. (2005) A case of osteoma with cholesteatoma in the external auditory canal. International Journal Auris Nasus, 32, 281-284. doi:10.1016/j.anl.2005.03.010

[9] Viswanatha, B. (2011) Characteristics of osteoma of the temporal bone in young adolescents. Ear, Nose \& Throat Journal, 90, 72-79. 